\title{
Visuospatial Asymmetries Arise from Differences in the Onset Time of Perceptual Evidence Accumulation
}

\author{
DDaniel P. Newman, ${ }^{1 *}$ Gerard M. Loughnane, ${ }^{2,3 *}$ Simon P. Kelly, ${ }^{5}$ Redmond G. 0’Connell, ${ }^{1,3,4}$ and Mark A. Bellgrove ${ }^{1,3}$ \\ ${ }^{1}$ Monash Institute for Cognitive and Clinical Neurosciences, School of Psychological Sciences, Monash University, Melbourne, Victoria 3800, Australia, \\ ${ }^{2}$ School of Engineering, ${ }^{3}$ Trinity College Institute of Neuroscience, and ${ }^{4}$ School of Psychology, Trinity College Dublin, Dublin 2, Ireland, and ${ }^{5}$ School of \\ Electrical and Electronic Engineering, University College Dublin, Dublin 4, Ireland
}

Healthy subjects tend to exhibit a bias of visual attention whereby left hemifield stimuli are processed more quickly and accurately than stimuli appearing in the right hemifield. It has long been held that this phenomenon arises from the dominant role of the right cerebral hemisphere in regulating attention. However, methods that would enable more precise understanding of the mechanisms underpinning visuospatial bias have remained elusive. We sought to finely trace the temporal evolution of spatial biases by leveraging a novel bilateral dot motion detection paradigm. In combination with electroencephalography, this paradigm enables researchers to isolate discrete neural signals reflecting the key neural processes needed for making these detection decisions. These include signals for spatial attention, early target selection, evidence accumulation, and motor preparation. Using this method, we established that three key neural markers accounted for unique between-subject variation in visuospatial bias: hemispheric asymmetry in posterior $\alpha$ power measured before target onset, which is related to the distribution of preparatory attention across the visual field; asymmetry in the peak latency of the early $\mathrm{N} 2 \mathrm{c}$ target-selection signal; and, finally, asymmetry in the onset time of the subsequent neural evidence-accumulation process with earlier onsets for left hemifield targets. Our development of a single paradigm to dissociate distinct processing components that track the temporal evolution of spatial biases not only advances our understanding of the neural mechanisms underpinning normal visuospatial attention bias, but may also in the future aid differential diagnoses in disorders of spatial attention.

Key words: asymmetry; decision making; EEG; evidence accumulation; pseudoneglect; spatial attention

\section{Significance Statement}

The significance of this research is twofold. First, it shows that individual differences in how humans direct their attention between left and right space reflects physiological differences in how early the brain starts to accumulate evidence for the existence of a visual target. Second, the novel methods developed here may have particular relevance to disorders of attention, such as unilateral spatial neglect. In the case of spatial neglect, pathological inattention to left space could have multiple underlying causes, including biased attention, impaired decision formation, or a motor deficit related to one side of space. Our development of a single paradigm to dissociate each of these components may aid in supporting more precise differential diagnosis in such heterogeneous disorders.

\section{Introduction}

It has long been held that visuospatial attention is lateralized to the right hemisphere (Bogen and Gazzaniga, 1965; Mesulam,

Received Nov. 14, 2016; revised Jan. 27, 2017; accepted Feb. 15, 2017.

Author contributions: D.P.N., G.M.L., R.G.O., and M.A.B. designed research; D.P.N. and G.M.L. performed research; D.P.N. and S.P.K. contributed unpublished reagents/analytic tools; D.P.N. and G.M.L. analyzed data; D.P.N., G.M.L., R.G.O., and M.A.B. wrote the paper.

This work was supported by grants from the Australian Research Council (FT130101488, DP150100986). We thank Hayley Pickering and Roisin White for their help with data collection.

*D.P.N. and G.M.L. contributed equally to this work.

The authors declare no competing financial interests.

Correspondence should be addressed to Mark A. Bellgrove at the above address. E-mail: mark.bellgrove@monash.edu. DOI:10.1523/JNEUROSCI.3512-16.2017

Copyright $\odot 2017$ the authors $\quad 0270-6474 / 17 / 373378-08 \$ 15.00 / 0$
1981; Young and Ratcliff, 1983; De Renzi, 1985). Primary evidence supporting this claim is that the neglect syndrome, which involves a profound spatial inattention for the contralesional hemifield, is more common, severe, and long-lasting following right compared with left hemisphere damage (Stone et al., 1993; Corbetta and Shulman, 2011). Subtle processing advantages for visuospatial stimuli presented to the left hemifield are also seen in most healthy individuals, a phenomenon known as "pseudoneglect” (Bowers and Heilman, 1980; Nicholls et al., 1999; Jewell and McCourt, 2000; Voyer et al., 2012). Recent evidence from genetics (Bellgrove et al., 2005, 2007, 2008, 2009; Newman et al., 2012; Zozulinsky et al., 2014) and structural, functional, and molecular brain imaging (Thiebaut de Schotten et al., 2011; Tomer et 
al., 2013; Chechlacz et al., 2015; Marshall et al., 2015) suggests that substantial individual difference factors contribute to visuospatial biases. Thiebaut de Schotten and colleagues reported that individual differences in the degree of visuospatial bias correlated with the extent of hemispheric asymmetry in white matter frontoparietal pathways, with larger volumes in the right, relative to left, associated with faster response times for left, compared with right, targets (Thiebaut de Schotten et al., 2011). One implication of this work is that individual differences in structural hemispheric asymmetries may facilitate faster processing of visuospatial stimuli presented to one or the other visual hemifield.

Perceptual decision making requires multiple neural-processing stages ranging from those for representing, selecting, and accumulating sensory information to those for preparing and executing actions. Visuospatial asymmetries can potentially reflect the contribution of any one of these processing stages, and thus the ability to measure each stage separately is important if we are to understand the neural processes underpinning normal and abnormal visuospatial biases. Recent developments in behavioral/ electroencephalographic (EEG) paradigms make it possible to measure discrete neural signals for each of these processing stages, which are each related to forthcoming behavioral performance. Such signals include those that distribute attention across hemifields and are measured via interhemispheric asymmetry in pretarget preparatory $\alpha$ power (Thut et al., 2006; Loughnane et al., 2015); those that enable early target selection and are measured in the form of the contralateral N2c component (Loughnane et al., 2016); those that support evidence accumulation and are measured via the centroparietal positivity (CPP), whose build-up rate predicts reaction time (RT) and scales with sensory evidence strength independent of sensory or motor demands (O'Connell et al., 2012; Kelly and O'Connell, 2013); and those that enable limb-selective motor preparation and are measured via contralateral $\beta$-band activity (Donner et al., 2009; O'Connell et al., 2012; de Lange et al., 2013).

Disruption to one or more of these stages when processing left or right hemifield targets could result in slower orienting and decision responses. The development of a single paradigm that can dissociate these neural signals within an individual and make it possible to track temporal evolution of spatial biases could have important theoretical and clinical benefits in terms of improved differential diagnoses in conditions where aberrant visuospatial attention indexes neurological vulnerability, such as unilateral spatial neglect (Corbetta and Shulman, 2011), attention-deficit/ hyperactivity disorder (ADHD; Voeller and Heilman, 1988; Sheppard et al., 1999; Geeraerts et al., 2008; Chan et al., 2009; Bellgrove et al., 2013), and dyslexia (Facoetti et al., 2001; Hari et al., 2001; Liddle et al., 2009; Stenneken et al., 2011).

Here, for the first time, we modeled individual differences in left versus right hemifield RT asymmetry as a function of asymmetries in neural signals representing each of the temporal stages of perceptual decision making to determine which signals explained unique variation in visuospatial bias among healthy individuals.

\section{Materials and Methods}

Participants. Data were collected from 80 (42 female) healthy righthanded volunteers, ages 18-37 years (mean, 23.2 years), reporting normal or corrected-to-normal vision, no history of neurological or psychiatric disorder, and no head injury resulting in loss of consciousness. Participants were recruited and tested under identical experimental protocols at either Monash University, Australia $(N=60)$, or Trinity College Dublin, Ireland $(N=20)$. The experimental protocol was approved by each university's human research ethics committee before testing, and carried out in accordance with the approved guidelines. Informed consent was obtained from all participants before testing.

Materials and task procedures. Participants were seated in a darkened room $56 \mathrm{~cm}$ from a 21 inch CRT $(85 \mathrm{~Hz}, 1024 \times 768$ resolution $)$ and asked to perform a variant of the random dot motion task (Newsome et al., 1989; Britten et al., 1992; Kelly and O'Connell, 2013; Loughnane et al., 2016). In this version of the task, participants fixated centrally and monitored two peripheral patches (one in each hemifield) of 150 moving dots for targets, which were defined by a seamless transition from random motion to coherent motion in a downward direction (Fig. 1A). Upon detecting a target, participants made a speeded button press with their right index finger. Peers et al. (2006), using a similar paradigm, found that the response hand had no effect on spatial bias. Targets were separated by intervals of random motion of 3.06, 5.17, or $7.29 \mathrm{~s}$ (randomized throughout each block). Participants completed 16 blocks consisting of 18 targets, each block lasting $\sim 3$ min. Participants were given a break of $\sim 30 \mathrm{~s}$ in between each block. An SR Research EyeLink eye tracker (EyeLink version 2.04, SR Research/SMI) recorded eye movements, which were later analyzed to ensure that participants maintained fixation. The center of each random dot motion patch was at a visual angle $10^{\circ}$ either side and $4^{\circ}$ below the fixation square; each patch covered $8^{\circ}$ visual angle and consisted of $1506 \times 6$ pixel white dots. The dot stimuli were flickered on and off the screen every $23.5 \mathrm{~ms}$, resulting in a flicker rate of 21.25 frames/s (each "frame" representing two screen refreshes). During random motion, these dots were placed randomly throughout the patch on each frame. During coherent motion, a proportion (50\%) of the dots was randomly selected on each frame to be displaced in the downward direction on the following frame, with a motion speed of $6^{\circ}$ per second. The paradigm was run using Matlab (MathWorks) and the Psychophysics Toolbox extensions (Brainard, 1997; Pelli, 1997; Cornelissen et al., 2002).

EEG acquisition and preprocessing. Continuous EEG was acquired from 64 scalp electrodes using an ActiveTwo system (Biosemi) digitized at $512 \mathrm{~Hz}$ (Trinity College Dublin data collection) or using a BrainAmp DC system (Brain Products) digitized at $500 \mathrm{~Hz}$ (Monash University data collection). Data were processed using a combination of custom scripts and EEGLAB (Delorme and Makeig, 2004) routines implemented in Matlab (MathWorks). All processing scripts used for the current study can be found on-line (https://github.com/gerontium/big_dots). A $35 \mathrm{~Hz}$ low-pass filter was applied off-line using a fourth-order Butterworth filter, noisy channels were interpolated (spherical spline), and the data were rereferenced to the average reference. Data from Trinity College Dublin and Monash University were combined by resampling the data collected in Dublin to $500 \mathrm{~Hz}$. Epochs were then extracted from the continuous data from -700 to $1800 \mathrm{~ms}$ around target onset, and baselined with respect to -100 to $0 \mathrm{~ms}$ before target onset.

Trials were excluded from analysis if any of the following conditions were met: (1) if RTs were $<150 \mathrm{~ms}$ (pre-emptive responses) or $>1800 \mathrm{~ms}$ (responses after coherent motion offset); (2) if the EEG from any channel exceeded $\pm 100 \mu \mathrm{V}$ during the interval from -500 to $0 \mathrm{~ms}$ before target onset for pretarget- $\alpha$ analysis, or during the interval from $-100 \mathrm{~ms}$ before target onset to $100 \mathrm{~ms}$ after RT for the ERP analysis; or (3) if central fixation was broken by blinking or eye movement $>3^{\circ}$ left or right of center, either from -500 to $0 \mathrm{~ms}$ before target onset for pretarget- $\alpha$ analysis or during the interval between $100 \mathrm{~ms}$ before target onset and $100 \mathrm{~ms}$ after response for the ERP analysis.

Power in the $\alpha$ band was calculated using temporal spectral evolution (TSE) methods, following Thut and colleagues (2006). Each epoch was bandpass filtered to $\alpha$ range $(8-13 \mathrm{~Hz}$ ), rectified (converted to absolute values), and trimmed to exclude the $200 \mathrm{~ms}$ at the beginning and end of the epoch to eliminate filter warm-up artifacts. Data were then smoothed by averaging inside a moving window of $100 \mathrm{~ms}$, moving forward in $50 \mathrm{~ms}$ increments. $\alpha$ Measurements were pooled from two symmetric parietal regions of interest (ROIs; left hemisphere: $\mathrm{CP} 5, \mathrm{CP} 3, \mathrm{P} 5, \mathrm{P} 3$; right hemisphere: CP4, CP6, P6, P8; Thut et al., 2006; ter Huurne et al., 2013). Pretarget- $\alpha$ power was defined as the mean power from -500 to $0 \mathrm{~ms}$ before target onset (Newman et al., 2016) and aggregated to participant means.

The N2c and N2i components were measured contralateral and ipsilateral to the target location, respectively, at electrodes P7 and P8 (Loughnane et al., 2016), while the CPP was measured at peak electrodes $\mathrm{Pz}$ 
A
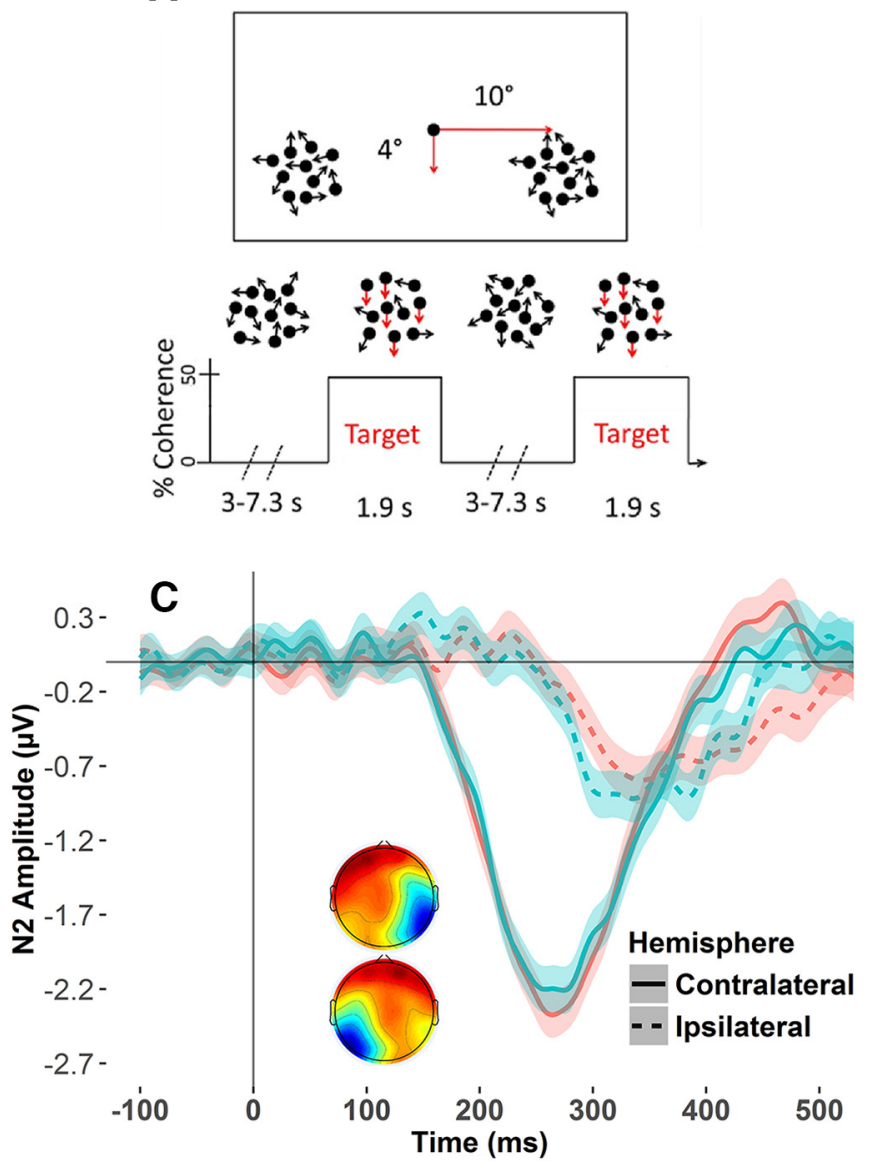
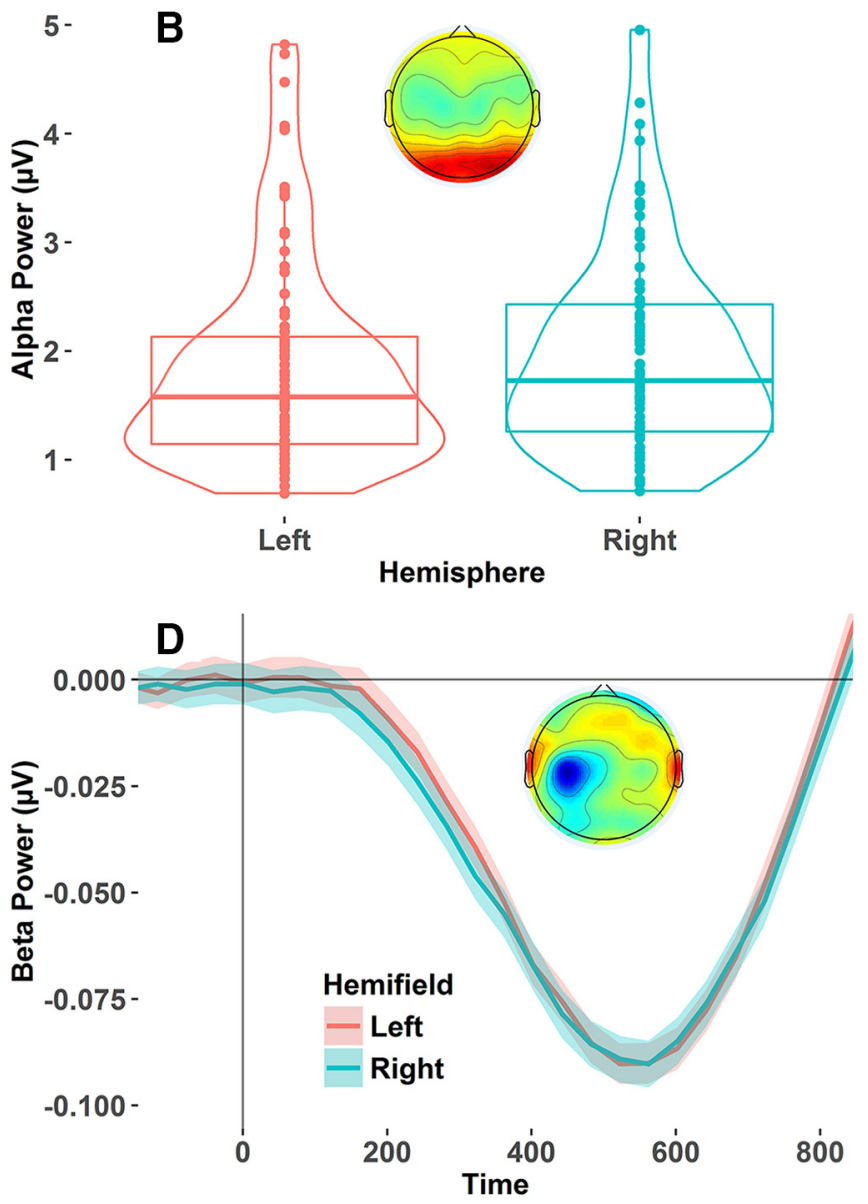

Figure 1. A, Behavioral paradigm. Participants fixated on the central dot while monitoring the two peripheral patches of randomly moving dots for intermittent periods of coherent downward motion that could occur in either the left or the right hemifield at an unpredictable time. Participants made a speeded button press upon detection of the coherent motion target. $\boldsymbol{B}$, Pretarget- $\alpha$ power was greater over right than left hemisphere posterior regions. C, Target Hemifield had no significant effect on the N2C or N2i signals. $\boldsymbol{D}$, Target Hemifield had no significant effect on motor selective LHB desynchronization. Insets throughout the subplots show scalp topography of each of the neural signals, and the error ribbons ( $\boldsymbol{C}$ and $\boldsymbol{D})$ represent $95 \%$ C for mean amplitude.

(O'Connell et al., 2012; Kelly and O'Connell, 2013; Murphy et al., 2015; Twomey et al., 2015; Loughnane et al., 2016).

The N2c, N2i, and CPP signals were aggregated to average waveforms as a function of target hemifield for each participant. N2 latency was then identified as the time point with the most negative amplitude value in the stimulus-locked waveform between 150 and $400 \mathrm{~ms}$ for the N2c and 200-450 ms for N2i, while N2 amplitude was measured as the mean amplitude inside a $100 \mathrm{~ms}$ window centered on the stimulus-locked grand average peak (N2c: 266 ms; N2i: 340 ms; Loughnane et al., 2016). $\mathrm{CPP}$ build-up rate was defined as the slope of a straight line fitted to the response-locked waveform (O'Connell et al., 2012; Kelly and O'Connell, 2013; Loughnane et al., 2016) with the time window defined individually for each participant as the $100 \mathrm{~ms}$ before the maximum CPP amplitude preresponse. Onset latency of the CPP was measured by performing running sample point by sample point $t$ tests against zero across each participant's stimulus-locked CPP waveforms. CPP onset was defined as the first point at which the amplitude reached significance at the 0.05 level for $\geq 15$ consecutive points (Foxe and Simpson, 2002; Kelly et al., 2008; Loughnane et al., 2016).

Left hemisphere $\beta$ power (LHB; $20-35 \mathrm{~Hz}$ ) was measured from the standard motor site C3, using short-time Fourier transform (STFT) with a $286 \mathrm{~ms}$ window size and $20 \mathrm{~ms}$ step size (O'Connell et al., 2012). The LHB signal was baselined with respect to -100 to $0 \mathrm{~ms}$ before target onset and aggregated to average waveforms as a function of the target hemifield for each participant. LHB amplitude was defined as the mean amplitude of the stimulus-locked waveform during the 300-500 ms window. The LHB build-up rate was defined as the slope of a straight line fitted to the response-locked waveform from -300 to $-50 \mathrm{~ms}$ before response. Note
STFT and TSE methods for measuring LHB and $\alpha$ power yielded highly comparable results.

Inferential analysis. Analysis of the effects of Hemisphere (left, right) on pretarget- $\alpha$ power, and of Target Hemifield (left, right) on the N2c/i, $\mathrm{CPP}, \beta$, and RT measures were conducted using repeated-measures ANOVA. In cases where the assumption of normality was violated, the $p$ value $\left(p_{\text {permuted }}\right)$ of a permutation test for the effect of Hemifield based on 1000 permutations is also reported.

To assess the extent that each of the neural signals (pretarget $\alpha, \mathrm{N} 2 \mathrm{c} / \mathrm{i}$, $\mathrm{CPP}, \beta$ ) could explain variation in visuospatial attention asymmetry between individuals, we calculated an index of each individual's visuospatial attention asymmetry as follows: RT asymmetry $=$ (left target RT - right target RT)/(left + right target RT). This index gives positive values when RTs are faster for right relative to the left targets (rightward spatial bias) and negative values when the opposite is true (leftward bias). Corresponding neural signal indices were also calculated for each of the target-evoked signals (i.e., N2c, CPP, and $\beta$ ) as follows: signal asymmetry = (left target signal right target signal)/(left + right target signal). A similar formula was used to calculate hemispheric asymmetry for pretarget parietal $\alpha$ power: $\alpha$ asymmetry $=($ right ROI $\alpha-$ left ROI $\alpha) /($ right + left ROI $\alpha)$. This index gives positive values when $\alpha$ activity is greater over the right hemisphere ROI relative to the left hemisphere ROI and negative values when $\alpha$ activity is greater over the left hemisphere ROI relative to the right. If no asymmetry exists then the index gives a zero value (for comparable use of asymmetry indices, see Thut et al., 2006).

After forcing the control variables (test location, sex, age) into a linear regression model predicting RT asymmetry, the neural signal asymmetry indices were each added sequentially into consecutive regression models 


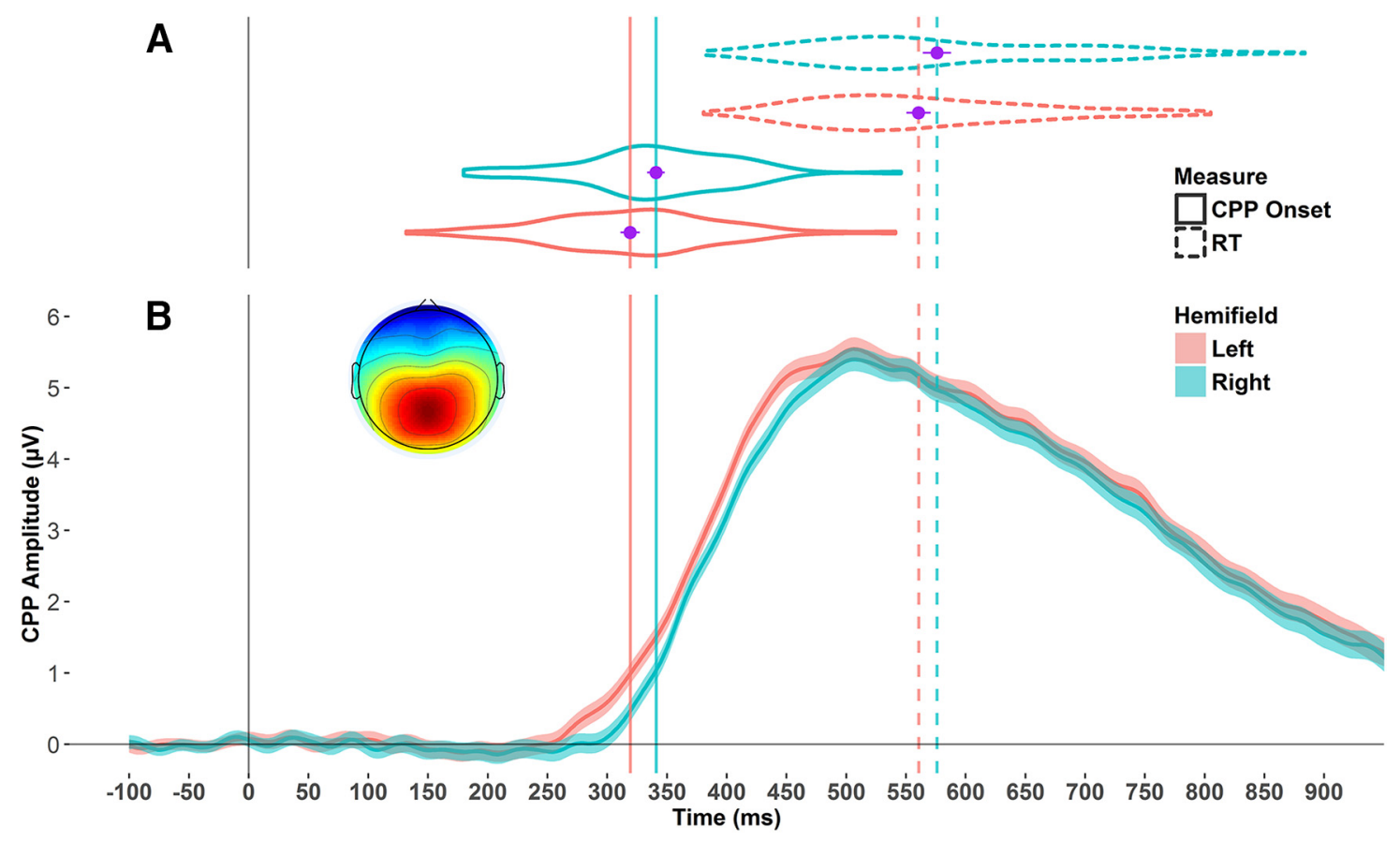

Figure 2. $\quad A$, CPP onset and RT both were significantly faster for left than right hemifield targets. Violin plots show conditional distributions while the purple dot-and-whisker plots show mean and SE. Vertical lines project the means down to B. B. Stimulus-locked CPP waveform shows earlier onset for left than right hemifield targets. Inset shows the scalp topography of the CPP. Error ribbons represent $95 \%$ Cl for mean amplitude.

in a hierarchical fashion with their entry order determined by their temporal order in the perceptual decision-making process. This allowed us to model individual differences in RT asymmetry, as a function of asymmetries in neural signals representing each temporal stage of perceptual decision making, from a pretarget preparatory signal ( $\alpha$ power), to target selection (N2c), to evidence accumulation (CPP), to motor preparation (LHB). The hierarchical entry method allowed us to assess whether each of the different signals improved the model fit for RT asymmetry over and above the signals that temporally precede it. Only those signals that explained unique variation in RT asymmetry were then selected for forced input into a final simplified RT-asymmetry model, which made it possible to obtain accurate parameter estimates not influenced by other signals shown not to improve model fit.

Analyses in the current manuscript focused on visuospatial biases at the level of individual participants, rather than within-participant absolute RT. Nevertheless, in accordance with past work, each of the neural signals in the current study was significantly correlated with absolute RT (e.g., pretarget $\alpha$ : Thut et al., 2006, Loughnane et al., 2015; the N2c component: Loughnane et al., 2016; the CPP: O'Connell et al., 2012; Kelly and O'Connell, 2013; limb-selective LHB: Donner et al., 2009; O'Connell et al., 2012; de Lange et al., 2013).

\section{Results}

Target-detection accuracy was at ceiling (mean, 98.7\%; range: $92-100 \%)$ and a factorial permutation test showed no effect of Target Hemifield on accuracy $(p=0.852)$. As expected, RTs were significantly faster for left hemifield targets (mean, $561 \mathrm{~ms} ; S D=$ 91) than right hemifield targets [mean, $576 \mathrm{~ms} ; S D=105$; $F_{(1,79)}=11.09, p=0.001, \eta_{G}^{2}$ (generalized $\eta$-squared $)=0.006$, $\left.p_{\text {permuted }}=0.001\right]$, which is characteristic of the phenomenon of pseudoneglect (Fig. 2A). A significant effect of Hemisphere showed that pretarget- $\alpha$ power was greater over the right (mean, $1.98 \mu \mathrm{V} ; S D=0.95$ ) compared with left parietal regions (mean, $1.85 \mu \mathrm{V} ; S D=0.97 ; F_{(1,79)}=11.47, p=0.001, \eta_{G}^{2}=0.004$, $p_{\text {permuted }}=0.001$; Fig. $1 B$ ). Although the N2c was noticeably larger following left hemifield targets, and the N2i larger following right hemifield targets, these differences did not produce sig- nificant main effects of Hemifield (N2i latency $F_{(1,79)}=0.15, p=$ $0.695, p_{\text {permuted }}=0.691 ; \mathrm{N} 2 \mathrm{i}$ amplitude $F_{(1,79)}=1.26, p=0.265$; $\mathrm{N} 2 \mathrm{c}$ latency $F_{(1,79)}=0.005, p=0.939, p_{\text {permuted }}=0.946 ; \mathrm{N} 2 \mathrm{c}$ amplitude $F_{(1,79)}=0.08, p=0.784$; Fig. $\left.1 C\right)$. Combining N2c and $\mathrm{N} 2 \mathrm{i}$ into a Hemisphere (contralateral, ipsilateral) $\times$ Hemifield (left, right) ANOVA confirmed that the contralateral signal is larger and earlier than the ipsilateral signal (amplitude $F_{(1,79)}=$ 62.74, $p<0.001, \eta_{G}^{2}=0.13$; latency $F_{(1,79)}=94.22, p<0.001$, $p_{\text {permuted }}<0.001, \eta_{G}^{2}=0.21$; Fig. $\left.1 C\right)$. However, there was no significant main effect of Hemifield (amplitude $F_{(1,79)}=0.40$, $p=0.528$; latency $F_{(1,79)}=0.16, p=0.695$, $\left.p_{\text {permuted }}=0.688\right)$ or Hemisphere $\times$ Hemifield interaction (amplitude $F_{(1,79)}=0.62$, $p=0.434$; latency $F_{(1,79)}=0.09, p=0.769$, $\left.p_{\text {permuted }}=0.743\right)$.

The CPP signal began significantly earlier for left hemifield targets (mean, $319 \mathrm{~ms} ; S D=72$ ) than for right hemifield targets (mean, $341 \mathrm{~ms} ; S D=65 ; F_{(1,79)}=5.54, p=0.025, \eta_{G}^{2}=0.025$ ), suggesting an earlier onset of the evidence accumulation process for left hemifield targets, consistent with the left hemifield RT advantage (Fig. $2 A, B$ ). There was, however, no effect of Hemifield on the build-up rate of the CPP signal $\left(F_{(1,79)}=1.26, p=\right.$ 0.266 ) or on the motor-selective LHB signal (LHB amplitude $F_{(1,79)}=0.68, p=0.413$, $p_{\text {permuted }}=0.418$; LHB slope $F_{(1,79)}=$ $0.19, p=0.661, p_{\text {permuted }}=0.66$; Fig. $\left.1 D\right)$.

Next, the neural signal asymmetry indices were each added to a model predicting RT asymmetry in a hierarchical fashion with their entry order determined by their temporal order in the perceptual decision-making process. This method allowed us to assess whether each of the different signals improved the model fit for RT asymmetry over and above the preceding signal. Note that none of the neural asymmetry predictors were highly correlated with each other.

When compared with an intercept-only model, the linear regression model including the control variables (test location, sex, age) offered no significant improvement in modeling RT asym- 
Table 1. Parameter estimates from the final linear regression model of RT asymmetry, which included only those neural asymmetry measures that improved model fit

\begin{tabular}{llllll}
\hline & $b$ (slope) & $b$ SE & $t$ & $\beta$ & $p$ \\
\hline Pretarget- $\alpha$ asymmetry & 0.093 & 0.048 & 1.934 & 0.201 & 0.057 \\
N2c-latency asymmetry & 0.066 & 0.036 & 1.859 & 0.192 & 0.067 \\
CPP-onset asymmetry & 0.080 & 0.026 & 3.014 & 0.313 & 0.004 \\
\hline
\end{tabular}

$\beta$ Symbolizes standardized $\beta$, which represents the relative importance of each predictor, independent of the unit of measurement. Together these three neural asymmetry measures explained $19 \%$ of the variation in RT asymmetry among participants.

metry $\left(R^{2}=0.042, F_{(3,76)}=1.27, p=0.29\right)$. The addition of pretarget- $\alpha$ asymmetry significantly improved model fit $\left(R^{2}=\right.$ $\left.0.086, R_{\text {change }}^{2}=0.044, F_{(1,75)}=4.02, p=0.048\right)$. The addition of $\mathrm{N} 2 \mathrm{c}$-amplitude asymmetry did not improve the model $\left(R^{2}=\right.$ $\left.0.086, R_{\text {change }}^{2}=0.00, F_{(1,74)}=0.01, p=0.918\right)$, while N2c-latency asymmetry provided a significant improvement $\left(R^{2}=0.133\right.$, $\left.R_{\text {change }}^{2}=0.047, F_{(1,73)}=4.30, p=0.041\right)$. Adding CPP-onset asymmetry also gave a significant improvement to the model $\left(R^{2}=0.21, R_{\text {change }}^{2}=0.08, F_{(1,72)}=7.43, p=0.008\right)$, while CPPslope asymmetry did not $\left(R^{2}=0.22, R_{\text {change }}^{2}=0.01, F_{(1,71)}=0.99\right.$, $p=0.324)$. Neither LHB-amplitude asymmetry $\left(R^{2}=0.23\right.$, $\left.R_{\text {change }}^{2}=0.01, F_{(1,70)}=0.84, p=0.363\right)$ nor LHB-slope asymmetry $\left(R^{2}=0.25, R_{\text {change }}^{2}=0.02, F_{(1,96)}=1.64, p=0.204\right)$ offered any improvement to the RT-asymmetry model. Overall, this hierarchical regression analysis shows that the pretarget- $\alpha$, N2clatency, and CPP-onset asymmetry indices each exert a partially independent influence on RT asymmetry.

An algorithm for forward/backward stepwise model selection using the Akaike information criterion (Venables and Ripley, 2002) supported the results from the hierarchical method, confirming that the only neural asymmetry signals to improve the model for RT asymmetry are pretarget- $\alpha$ asymmetry, N2clatency asymmetry, and CPP-onset asymmetry. Thus, these three signals only were then forced into a final linear regression model of RT asymmetry $\left(R^{2}=0.19, F_{(3,76)}=5.82, p=0.001\right)$ and their resulting parameter estimates are shown in Table 1 below. This simplified model was necessary to obtain accurate parameter estimates for pretarget- $\alpha, \mathrm{N} 2 \mathrm{c}$-latency, and CPP-onset asymmetry that are not influenced by the other, noninformative, signals.

Table 1 shows pretarget- $\alpha$ asymmetry $(p=0.057)$ and N2clatency asymmetry $(p=0.067)$ fell short of the nominal statistical significance threshold for their effect on RT asymmetry when forced into the final linear regression model. This result is ambiguous since it conflicts with the results of the hierarchical regression method used above, which found that, along with CPP-onset asymmetry, both pretarget- $\alpha$ and N2c-latency asymmetry added significant improvement to the RT-asymmetry model. Likewise, the forward/backwards stepwise method selected pretarget $\alpha$, $\mathrm{N} 2 \mathrm{c}$ latency, and CPP onset as the best predictors for the model. Therefore, to confirm whether each of the three neural asymmetry measures had a significant effect on RT asymmetry, we performed "robust regression" based on 5000 bootstrap replicates to calculate the $95 \%$ CI around slope estimates for the final model (Table 2).

The robust regression (Table 2) confirmed that pretarget- $\alpha$ asymmetry, N2c-latency asymmetry, and CPP-onset asymmetry all have a significant positive relationship with RT asymmetry. These effects are in the expected direction based on past research (Thut et al., 2006; Loughnane et al., 2015). The positive relationship between pretarget- $\alpha$ asymmetry and RT asymmetry (Fig. $3 A$ ) indicates that those participants with more positive pretarget- $\alpha$ asymmetry (i.e., relatively greater pretarget- $\alpha$

Table 2. Robust regression analysis

Bootstrapped 95\% Cls for b (slope) estimates ${ }^{a}$

Pretarget- $\alpha$ asymmetry

N2c-latency asymmetry

CPP-onset asymmetry

$0.003-0.180$

$0.001-0.149$

$0.025-0.147$

${ }^{a}$ Bootstrapped $95 \%$ confidence intervals (Cls) for the slope estimate of the neural signals in the final linear regression model of RT asymmetry.

power over their right than left hemisphere) tended to have more positive RT asymmetry (i.e., relatively slower RTs for left hemifield targets or relatively faster RTs for right hemifield targets). The positive relationship between N2c-latency asymmetry and RT asymmetry (Fig. 3B) indicates that participants with more negative N2c-latency asymmetry (i.e., earlier N2c latency for left than right hemifield targets) also tended to have more negative RT asymmetry (i.e., tended to respond relatively faster to left than right hemifield targets). Finally, CPP-onset asymmetry had the strongest relationship with RT asymmetry (Tables 1,2 ). This positive relationship (Fig. $3 C$ ) indicates that participants who had more leftward CPP-onset bias (i.e., CPP onset relatively earlier for left than right hemifield targets) also tended to have more prominent leftward RT asymmetry (i.e., tended to respond faster to left than right hemifield targets). This result suggests that earlier onset of the evidence accumulation process for left hemifield targets leads to a left hemifield RT advantage. That CPP-onset asymmetry was the strongest predictor of RT asymmetry is not due solely to its temporal proximity to response execution. This is the case since neither CPP slope nor LHB asymmetry, which occur closer to response, explained unique variance in RT asymmetry.

\section{Discussion}

This study asked whether individual differences in visuospatial asymmetry are indexed by functional asymmetries in electrocortical activity. We used a perceptual decision-making framework, with bilaterally presented dot motion targets, to track the temporal evolution of discrete EEG signals contributing to visuospatial biases. In line with previous reports, we found that hemispheric differences in pretarget posterior $\alpha$-band activity accounted for individual differences in visuospatial bias. But further to this, we also uncovered independent contributions from target-selection and evidence-accumulation signals that have not been previously reported. An asymmetry index derived from the peak latency of the N2c, a target-selection signal, explained unique variation in visuospatial bias among individuals. The CPP signal, which tracks perceptual evidence accumulation, began earlier for left than for the right hemifield targets and the degree of this asymmetry also explained individual differences in visuospatial bias.

Previous electrophysiology work on visuospatial attention has focused on hemispheric asymmetries in pretarget- $\alpha$ power (Worden et al., 2000; Sauseng et al., 2005; Thut et al., 2006; Gould et al., 2011; Newman et al., 2013; ter Huurne et al., 2013; Capilla et al., 2014; Loughnane et al., 2015). These studies have shown that processing advantages for the left relative to the right hemifield, particularly in cued target-detection paradigms, are related to more desynchronization of $\alpha$ over right, relative to left, posterior regions. We support this finding, albeit with a relatively small effect size, in the context of an uncued fixation-controlled paradigm. Although absolute $\alpha$ power was greater over the right posterior hemisphere at the group grand average (Slagter et al., 2016), individual differences in the hemispheric $\alpha$-asymmetry index related to RT asymmetry in the direction expected. The 

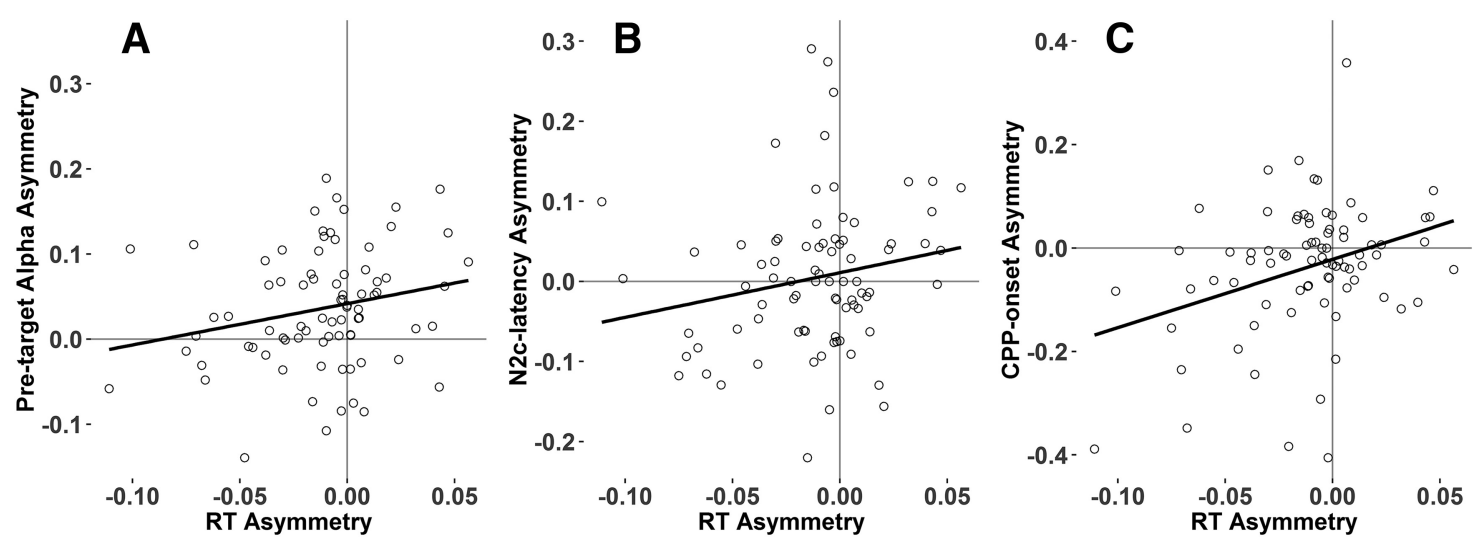

Figure 3. Pretarget- $\alpha, \mathrm{N} 2 c$-latency, and (PP-onset asymmetry indices each exert a partially independent influence on RT asymmetry. $\boldsymbol{A}$, The positive relationship indicates that those participants with relatively greater pretarget- $\alpha$ power over their right than over their left hemisphere also tended to have relatively slower RTs for left hemifield targets than for right hemifield targets. $B$, Participants with earlier N2c latency for left than for right hemifield targets also tended to have relatively faster RTs to left than to right hemifield targets. C, Participants who had earlier CPP onsets for left than right hemifield targets also tended to have faster RTs for left than right hemifield targets. Note that the relationships shown in $\boldsymbol{A}$ and $\boldsymbol{B}$ are of small effect size. However, a "robust regression analysis" confirmed that their slope is significantly $>0$ (Table 2).

positive relationship between $\alpha$ and RT asymmetry indicates that individuals with relatively less spontaneous pretarget- $\alpha$ power over their right than left hemisphere tended to have relatively faster RTs for left than right hemifield targets, as expected. It is interesting therefore that the grand averages show greater right hemisphere $\alpha$ and faster left hemifield RTs at the group level. It is possible that this is caused by a separate source of right hemisphere $\alpha$ modulation, which is not related to spatial bias and which merges on the scalp with the signal produced by $\alpha$ generators for spatial attention.

The absence of sudden stimulus intensity changes in the current paradigm eliminated visual-evoked transients from the event-related EEG trace. This helped provide after target onset a clearer view of the neural signals that contribute to decision formation, increasing their signal-to-noise ratio and aiding in their correlation with behavior. On the other hand, transient stimuli (e.g., a checkerboard appearing on screen) can elicit the P1 component and other visual evoked potentials than occur earlier those recorded in the current study. Those visual evoked potentials are modulated by spatial attention and reflect activity of both striate and extrastriate regions (Robinson and Rugg, 1988; Luck, 1995; Clark and Hillyard, 1996; Di Russo et al., 2002, 2008). Thus, the measurement of such signals may also prove informative for investigating stimulus-driven asymmetries in spatial-attention bias.

Recently, Loughnane et al. (2016) used this paradigm to demonstrate that early target-selection signals reliably precede the onset of the neural evidence-accumulation process during perceptual decision formation. The N2 selection signal, which consists of distinct contralateral and ipsilateral components, was found to predict behavioral performance (RT and accuracy) and the build-up rate of the CPP evidence-accumulation signal, even in the absence of distractor stimuli (Loughnane et al., 2016). Our novel finding that those with relatively earlier N2c latency over their right than left hemispheres also tend to have relatively faster RTs for left than right hemifield targets suggests that the speed of contralateral target-selection processes is a factor in individual differences in spatial bias.

CPP onset, which began earlier for left than for right hemifield targets, showed a clear effect of Target Hemifield. Furthermore, the left versus right hemifield asymmetry index for CPP onset showed the strongest relationship with RT asymmetry. The CPP signal was recently characterized as a domain-general signal representing a build-to-threshold decision variable, with dynamics that predict the timing and accuracy of perceptual reports independently of specific sensory or motor requirements (O'Connell et al., 2012; Kelly and O'Connell, 2013; Murphy et al., 2015; Twomey et al., 2015; Loughnane et al., 2016). Here we build on mechanistically principled characterization of the relevant signals to investigate neural asymmetry indices and thereby advance our understanding of neural correlates of visuospatial bias. Since all of the neural signals used here are robustly related to absolute RTs, it is informative that only a subset of their asymmetry indexes was related to individual differences in RT asymmetry.

The strongest of these indexes was CPP onset, which reflects the onset timing of an evidence-accumulation process ( $\mathrm{O}^{\prime} \mathrm{Connell} \mathrm{et} \mathrm{al.,}$ 2012; Kelly and O'Connell, 2013; Loughnane et al., 2016; Twomey et al., 2016). The significantly earlier CPP onset for left hemifield targets indicates that the evidence-accumulation process tends to start earlier for those targets, and this likely facilitates the improved processing of left compared with right hemifield targets, commonly described as pseudoneglect (Bowers and Heilman, 1980; Nicholls et al., 1999; Jewell and McCourt, 2000; Voyer et al., 2012). Although the CPP peaks over the central parietal scalp area, its dynamics bear a striking similarity to neural firing rate signals recorded in lateral parietal areas, such as the lateral intraparietal cortex, during primate neurophysiology studies using similar visual stimuli (Shadlen and Newsome, 2001). Since the right parietal cortex is heavily implicated in visuospatial attention in humans (Corbetta and Shulman, 2011), it will be of interest for future perceptual decision work to use combined EEG/fMRI to explore the anatomical source of the CPP signal in humans.

Previous studies have found that contralateral limb-selective $\beta$ activity, before a motor response, shows similar build-tothreshold dynamics as the CPP (Donner et al., 2009; O'Connell et al., 2012; de Lange et al., 2013). These dynamics in the $\beta$ signal are only seen when a motor response is required, whereas the CPP represents perceptual decision formation independently of specific motor requirements (O'Connell et al., 2012). Here the LHB signal did not differ between left and right hemifield targets and did not relate to individual differences in visuospatial bias. That $\mathrm{CPP}$ onset was related to the target hemifield and to individual differences in visuospatial bias, while LHB was not, may be due to the poorer temporal resolution of the LHB power measure, com- 
pared with the high resolution of CPP amplitude. Future work should counterbalance the response hand between blocks, within individual participants, to allow the measurement of a broadband lateralized readiness potential index (Gratton et al., 1988; de Jong et al., 1988; Eimer, 1998), which would have the same high temporal resolution as the CPP.

It should be noted that the exact results of the current study can only be generalized to the healthy right-handed population (the 90\% majority; Sun and Walsh, 2006). This was, however, a deliberate experimental control. Studies of lateralization of function have shown that although handedness varies naturally in the population, left-handedness can sometimes be determined by early developmental effects (Gutwinski et al., 2011; Willems et al., 2014). Consequently, including a left-handed cohort would necessitate substantial increases in the sample size and measures to ensure that left-handedness was not related to developmental differences. Therefore, this study was restricted to right-handed participants, in line with most other studies of spatial attention and with cognitive neuroscience studies more generally (Willems et al., 2014).

Although applied to healthy right-handed adults, the general approach described here may have important implications for studying disorders involving abnormal spatial-orienting biases. In the case of unilateral spatial neglect, for example, pathological inattention to left space could have multiple underlying causes, including biased attention, impaired decision formation, or a motor deficit related to one side of space. Yet current clinical tests for spatial neglect rely on classic paper-and-pencil tests, such as line bisection or cancellation tasks, which confound sensory, attentional, and motoric processes. Our development of a single electrophysiology paradigm that can dissociate each of these components to track the temporal evolution of spatial biases may aid differential diagnosis in such heterogeneous disorders of attention. An unprecedented level of specificity regarding deficient processes may yet be gained in future research by applying these methods to disorders involving aberrant visuospatial biases, such as unilateral spatial neglect, ADHD, and dyslexia (Voeller and Heilman, 1988; Sheppard et al., 1999; Facoetti et al., 2001; Hari et al., 2001; Geeraerts et al., 2008; Chan et al., 2009; Liddle et al., 2009; Corbetta and Shulman, 2011; Stenneken et al., 2011).

\section{Notes}

Supplemental material for this article is available at https://figshare.com/articles/Supplemental_Material_Visuospatial_asymmetries_arise_from_differences_in_the_onset_time_of_percep tual_evidence_accumulation/4591027. In accordance with past work, each of the neural signals in the current study was significantly correlated with absolute RTs (Supplemental Fig. 1). Note that none of the neural asymmetry predictors were highly correlated with each other (Supplemental Fig. 2). This material has not been peer reviewed. Analysis scripts and paradigm code (https://github.com/gerontium/big_dots) as well as the raw data (https://figshare.com/s/8d6f461834c47180a444) are open access and available under a Creative Commons AttributionNonCommercial-ShareAlike 3.0 International License.

\section{References}

Bellgrove MA, Hawi Z, Kirley A, Fitzgerald M, Gill M, Robertson IH (2005) Association between dopamine transporter (DAT1) genotype, left-sided inattention, and an enhanced response to methylphenidate in attention-deficit hyperactivity disorder. Neuropsychopharmacology 30:2290-2297. CrossRef Medline

Bellgrove MA, Chambers CD, Johnson KA, Daibhis A, Daly M, Hawi Z, Lambert D, Gill M, Robertson IH (2007) Dopaminergic genotype biases spatial attention in healthy children. Mol Psychiatry 12:786-792. CrossRef Medline

Bellgrove MA, Barry E, Johnson KA, Cox M, Daibhis A, Daly M, Hawi Z, Lambert D, Fitzgerald M, McNicholas F, Robertson IH, Gill M, Kirley A, Dáibhis A (2008) Spatial attentional bias as a marker of genetic risk, symptom severity, and stimulant response in ADHD. Neuropsychopharmacology 33:2536-2545. CrossRef Medline

Bellgrove MA, Johnson KA, Barry E, Mulligan A, Hawi Z, Gill M, Robertson I, Chambers CD (2009) Dopaminergic haplotype as a predictor of spatial inattention in children with attention-deficit/hyperactivity disorder. Arch Gen Psychiatry 66:1135-1142. CrossRef Medline

Bellgrove MA, Eramudugolla R, Newman DP, Vance A, Mattingley JB (2013) Influence of attentional load on spatial attention in acquired and developmental disorders of attention. Neuropsychologia 51:1085-1093. CrossRef Medline

Bogen JE, Gazzaniga MS (1965) Cerebral commissurotomy in man: minor hemisphere dominance for certain visuo-spatial functions. J Neurosurg 23:394-399. CrossRef

Bowers D, Heilman KM (1980) Pseudoneglect: effects of hemispace on a tactile line bisection task. Neuropsychologia 18:491-498. CrossRef Medline

Brainard DH (1997) The Psychophysics Toolbox. Spat Vis 10:433-436. CrossRef Medline

Britten KH, Shadlen MN, Newsome WT, Movshon JA (1992) The analysis of visual motion: a comparison of neuronal and psychophysical performance. J Neurosci 12:4745-4765. Medline

Capilla A, Schoffelen JM, Paterson G, Thut G, Gross J (2014) Dissociated $\alpha$-band modulations in the dorsal and ventral visual pathways in visuospatial attention and perception. Cereb Cortex 24:550-561. CrossRef Medline

Chan E, Mattingley JB, Huang-Pollock C, English T, Hester R, Vance A, Bellgrove MA (2009) Abnormal spatial asymmetry of selective attention in ADHD. J Child Psychol Psychiatry 50:1064-1072. CrossRef Medline

Chechlacz M, Gillebert CR, Vangkilde SA, Petersen A, Humphreys GW (2015) Structural variability within frontoparietal networks and individual differences in attentional functions: an approach using the theory of visual attention. J Neurosci 35:10647-10658. CrossRef Medline

Clark VP, Hillyard SA (1996) Spatial selective attention affects early extrastriate but not striate components of the visual evoked potential. J Cogn Neurosci 8:387-402. CrossRef Medline

Corbetta M, Shulman GL (2011) Spatial neglect and attention networks. Annu Rev Neurosci 34:569-599. CrossRef Medline

Cornelissen FW, Peters EM, Palmer J (2002) The Eyelink Toolbox: eye tracking with MATLAB and the Psychophysics Toolbox. Behav Res Methods Instrum Comput 34:613-617. CrossRef Medline

De Renzi E (1985) Disorders of space, exploration and cognition. New York: Wiley.

de Jong R, Wierda M, Mulder G, Mulder LJ (1988) Use of partial stimulus information in response processing. J Exp Psychol Hum Percept Perform 14:682-692. CrossRef Medline

de Lange FP, Rahnev DA, Donner TH, Lau H (2013) Prestimulus oscillatory activity over motor cortex reflects perceptual expectations. J Neurosci 33:1400-1410. CrossRef Medline

Delorme A, Makeig S (2004) EEGLAB: an open source toolbox for analysis of single-trial EEG dynamics including independent component analysis. J Neurosci Methods 134:9-21. CrossRef Medline

Di Russo F, Martínez A, Sereno MI, Pitzalis S, Hillyard SA (2002) Cortical sources of the early components of the visual evoked potential. Hum Brain Mapp 15:95-111. CrossRef Medline

Di Russo F, Aprile T, Spitoni G, Spinelli D (2008) Impaired visual processing of contralesional stimuli in neglect patients: a visual-evoked potential study. Brain 131:842-854. CrossRef Medline

Donner TH, Siegel M, Fries P, Engel AK (2009) Buildup of choicepredictive activity in human motor cortex during perceptual decision making. Curr Biol 19:1581-1585. CrossRef Medline

Eimer M (1998) The lateralized readiness potential as an on-line measure of central response activation processes. Behav Res Methods Instruments Comput 30:146-156. CrossRef

Facoetti A, Turatto M, Lorusso ML, Mascetti GG (2001) Orienting of visual attention in dyslexia: evidence for asymmetric hemispheric control of attention. Exp Brain Res 138:46-53. CrossRef Medline

Foxe JJ, Simpson GV (2002) Flow of activation from V1 to frontal cortex in 
humans: a framework for defining "early" visual processing. Exp Brain Res 142:139-150. CrossRef Medline

Geeraerts S, Lafosse C, Vaes N, Vandenbussche E, Verfaillie K (2008) Dysfunction of right-hemisphere attentional networks in attention deficit hyperactivity disorder. J Clin Exp Neuropsychol 30:42-52. Medline

Gould IC, Rushworth MF, Nobre AC (2011) Indexing the graded allocation of visuospatial attention using anticipatory alpha oscillations. J Neurophysiol 105:1318-1326. CrossRef Medline

Gratton G, Coles MG, Sirevaag EJ, Eriksen CW, Donchin E (1988) Pre- and poststimulus activation of response channels: a psychophysiological analysis. J Exp Psychol Hum Percept Perform 14:331-344. CrossRef Medline

Gutwinski S, Löscher A, Mahler L, Kalbitzer J, Heinz A, Bermpohl F (2011) Understanding left-handedness. Dtsch Arztebl Int 108:849-853. CrossRef Medline

Hari R, Renvall H, Tanskanen T (2001) Left minineglect in dyslexic adults. Brain 124:1373-1380. CrossRef Medline

Jewell G, McCourt ME (2000) Pseudoneglect: a review and meta-analysis of performance factors in line bisection tasks. Neuropsychologia 38:93-110. CrossRef Medline

Kelly SP, O'Connell RG (2013) Internal and external influences on the rate of sensory evidence accumulation in the human brain. J Neurosci 33: 19434-19441. CrossRef Medline

Kelly SP, Gomez-Ramirez M, Foxe JJ (2008) Spatial attention modulates initial afferent activity in human primary visual cortex. Cereb Cortex 18:2629-2636. CrossRef Medline

Liddle EB, Jackson GM, Rorden C, Jackson SR (2009) Lateralized temporal order judgement in dyslexia. Neuropsychologia 47:3244-3254. CrossRef Medline

Loughnane GM, Shanley JP, Lalor EC, O’Connell RG (2015) Behavioral and electrophysiological evidence of opposing lateral visuospatial asymmetries in the upper and lower visual fields. Cortex 63:220-231. CrossRef Medline

Loughnane GM, Newman DP, Bellgrove MA, Lalor EC, Kelly SP, O'Connell RG (2016) Target selection signals influence perceptual decisions by modulating the onset and rate of evidence accumulation. Curr Biol 26: 496-502. CrossRef Medline

Luck SJ (1995) Multiple mechanisms of visual-spatial attention: recent evidence from human electrophysiology. Behav Brain Res 71:113-123. Medline

Marshall TR, Bergmann TO, Jensen O (2015) Frontoparietal structural connectivity mediates the top-down control of neuronal synchronization associated with selective attention. PLOS Biol 13:e1002272. CrossRef Medline

Mesulam MM (1981) A cortical network for directed attention and unilateral neglect. Ann Neurol 10:309-325. Medline

Murphy PR, Robertson IH, Harty S, O'Connell RG (2015) Neural evidence accumulation persists after choice to inform metacognitive judgments. Elife pii:e11946. CrossRef Medline

Newman DP, O'Connell RG, Nathan PJ, Bellgrove MA (2012) Dopamine transporter genotype predicts attentional asymmetry in healthy adults. Neuropsychologia 50:2823-2829. CrossRef Medline

Newman DP, O’Connell RG, Bellgrove MA (2013) Linking time-on-task, spatial bias and hemispheric activation asymmetry: a neural correlate of rightward attention drift. Neuropsychologia 51:1215-1223. CrossRef Medline

Newman DP, Lockley SW, Loughnane GM, Martins AC, Abe R, Zoratti MT, Kelly SP, O’Neill MH, Rajaratnam SM, O'Connell RG, Bellgrove MA (2016) Ocular exposure to blue-enriched light has an asymmetric influence on neural activity and spatial attention. Sci Rep 6:27754. CrossRef Medline

Newsome WT, Britten KH, Movshon JA (1989) Neuronal correlates of a perceptual decision. Nature 341:52-54. CrossRef Medline

Nicholls ME, Bradshaw JL, Mattingley JB (1999) Free-viewing perceptual asymmetries for the judgement of brightness, numerosity and size. Neuropsychologia 37:307-314. CrossRef Medline

O'Connell RG, Dockree PM, Kelly SP (2012) A supramodal accumulationto-bound signal that determines perceptual decisions in humans. Nat Neurosci 15:1729-1735. CrossRef Medline

Peers PV, Cusack R, Duncan J (2006) Modulation of spatial bias in the dual task paradigm: evidence from patients with unilateral parietal lesions and controls. Neuropsychologia 44:1325-1335. CrossRef Medline

Pelli DG (1997) The VideoToolbox software for visual psychophysics: transforming numbers into movies. Spat Vis 10:437-442. Medline

Robinson DL, Rugg MD (1988) Latencies of visually responsive neurons in various regions of the rhesus monkey brain and their relation to human visual responses. Biol Psychol 26:111-116. Medline

Sauseng P, Klimesch W, Stadler W, Schabus M, Doppelmayr M, Hanslmayr S, Gruber WR, Birbaumer N (2005) A shift of visual spatial attention is selectively associated with human EEG alpha activity. Eur J Neurosci 22:2917-2926. Medline

Shadlen MN, Newsome WT (2001) Neural basis of a perceptual decision in the parietal cortex (area LIP) of the rhesus monkey. J Neurophysiol 86: 1916-1936. Medline

Sheppard DM, Bradshaw JL, Mattingley JB, Lee P (1999) Effects of stimulant medication on the lateralisation of line bisection judgements of children with attention deficit hyperactivity disorder. J Neurol Neurosurg Psychiatry 66:57-63. Medline

Slagter HA, Prinssen S, Reteig LC, Mazaheri A (2016) Facilitation and inhibition in attention: functional dissociation of pre-stimulus alpha activity, P1, and N1 components. Neuroimage 125:25-35. CrossRef Medline

Stenneken P, Egetemeir J, Schulte-Körne G, Müller HJ, Schneider WX, Finke K (2011) Slow perceptual processing at the core of developmental dyslexia: a parameter-based assessment of visual attention. Neuropsychologia 49:3454-3465. CrossRef Medline

Stone SP, Halligan PW, Greenwood RJ (1993) The incidence of neglect phenomena and related disorders in patients with an acute right or left hemisphere stroke. Age Ageing 22:46-52. Medline

Sun T, Walsh CA (2006) Molecular approaches to brain asymmetry and handedness. Nat Rev Neurosci 7:655-662. Medline

ter Huurne N, Onnink M, Kan C, Franke B, Buitelaar J, Jensen O (2013) Behavioral consequences of aberrant alpha lateralization in attentiondeficit/hyperactivity disorder. Biol Psychiatry 74:227-233. CrossRef Medline

Thiebaut de Schotten M, Dell'Acqua F, Forkel SJ, Simmons A, Vergani F, Murphy DG, Catani M (2011) A lateralized brain network for visuospatial attention. Nat Neurosci 14:1245-1246. CrossRef Medline

Thut G, Nietzel A, Brandt SA, Pascual-Leone A (2006) $\alpha$-Band electroencephalographic activity over occipital cortex indexes visuospatial attention bias and predicts visual target detection. J Neurosci 26:9494-9502. CrossRef Medline

Tomer R, Slagter HA, Christian BT, Fox AS, King CR, Murali D, Davidson RJ (2013) Dopamine asymmetries predict orienting bias in healthy individuals. Cereb Cortex 23:2899-2904. CrossRef Medline

Twomey DM, Murphy PR, Kelly SP, O’Connell RG (2015) The classic P300 encodes a build-to-threshold decision variable. Eur J Neurosci 42:16361643. CrossRef Medline

Twomey DM, Kelly SP, O’Connell RG (2016) Abstract and effectorselective decision signals exhibit qualitatively distinct dynamics before delayed perceptual reports. J Neurosci 36:7346-7352. CrossRef Medline

Venables WN, Ripley BD (2002) Modern Applied Statistics with S (4th ed.). New York: Springer.

Voeller KK, Heilman KM (1988) Attention deficit disorder in children: a neglect syndrome? Neurology 38:806-808. CrossRef Medline

Voyer D, Voyer SD, Tramonte L (2012) Free-viewing laterality tasks: a multilevel meta-analysis. Neuropsychology 26:551-567. CrossRef Medline

Willems RM, Van der Haegen L, Fisher SE, Francks C (2014) On the other hand: including left-handers in cognitive neuroscience and neurogenetics. Nat Rev Neurosci 15:193-201. CrossRef Medline

Worden MS, Foxe JJ, Wang N, Simpson GV (2000) Anticipatory biasing of visuospatial attention indexed by retinotopically specific alpha-band electroencephalography increases over occipital cortex. J Neurosci 20:RC63. Medline

Young AW, Ratcliff G (1983) Visuospatial abilities of the right hemisphere. In: Functions of the right cerebral hemisphere (Young AW, ed). London: Academic.

Zozulinsky P, Greenbaum L, Brande-Eilat N, Braun Y, Shalev I, Tomer R (2014) Dopamine system genes are associated with orienting bias among healthy individuals. Neuropsychologia 62:48-54. CrossRef Medline 